Nota científica

\title{
Nuevo registro y ampliación de la distribución del género Thylamys (Didelphimorphia: Didelphidae) en la región pampeana de Argentina
}

\author{
New record and distributional range extension of Thylamys (Didelphimorphia: Didelphidae) in the \\ Pampas region of Argentina
}

\author{
Oscar A. Stellatelli* y Alejandro V. Baladrón \\ Laboratorio de Vertebrados, Instituto de Investigaciones Marinas y Costeras, Consejo Nacional de Investigaciones Científicas y Técnicas, \\ Universidad Nacional de Mar del Plata. Funes 3350, Mar del Plata (B7602AYJ), Argentina
}

Recibido el 23 de mayo de 2014; aceptado el 8 de diciembre de 2014

Disponible en Internet el 26 de mayo de 2015

\begin{abstract}
Resumen
Se documenta un nuevo registro para el género de comadrejas enanas Thylamys, con base en una hembra capturada en el Partido de General Pueyrredón, provincia de Buenos Aires, Argentina. El individuo fue encontrado en un ambiente serrano correspondiente al sistema de Tandilia, uno de los 2 sistemas de sierras de la región pampeana. Este ejemplar representa el registro más oriental, expandiendo su distribución $135 \mathrm{~km}$ hacia el este. El ejemplar fue liberado in situ luego de que se tomaran las medidas corporales y fotografías para su identificación. Se requieren futuros análisis craneométricos y genéticos sobre la población, a fin de confirmar si corresponde a Thylamys pallidior, especie que cuenta con el registro de distribución más cercano a nuestro sitio de estudio.

Derechos Reservados ( 2015 Universidad Nacional Autónoma de México, Instituto de Biología. Este es un artículo de acceso abierto distribuido bajo los términos de la Licencia Creative Commons CC BY-NC-ND 4.0.
\end{abstract}

Palabras clave: Marmosa; Provincia de Buenos Aires; Sistema serrano de Tandilia

\begin{abstract}
A new record for the desert mouse opossum of the genus Thylamys is reported. One female was captured in the General Pueyrredón County, Buenos Aires Province, Argentina. The specimen was found in a mountain environment corresponding to Tandilia system, one of the 2 mountain systems of the Pampas Region. This specimen constitutes the easternmost record, extending its distribution range $135 \mathrm{~km}$ eastwards. The specimen was released in situ after being measured and photographed for further identification. Future craniometric and genetic analyses are needed to confirm whether it correspond to Thylamys pallidior, the species that has the closest record to our study site.

All Rights Reserved (C) 2015 Universidad Nacional Autónoma de México, Instituto de Biología. This is an open access item distributed under the
\end{abstract} Creative Commons CC License BY-NC-ND 4.0.

Keywords: Desert mouse opossum; Buenos Aires province; Tandilia mountain system

El género Thylamys incluye un conjunto de marsupiales de tamaño pequeño conocidos comúnmente como marmosas o comadrejas enanas, las cuales se caracterizan por tener hábitos nocturnos e insectívoros, carecer de marsupio y por su capacidad de acumular grasa en la cola (Gardner, 2007). Este

\footnotetext{
* Autor para correspondencia.

Correo electrónico: os2830@gmail.com (O.A. Stellatelli).

La revisión por pares es responsabilidad de la Universidad Nacional Autónoma de México.
}

género es endémico de Sudamérica y es uno de los pocos grupos de marsupiales asociados a ambientes áridos o semiáridos en esta región. Si bien durante la última década se han realizado numerosos trabajos para entender la sistemática, distribución y biogeografía de Thylamys (Braun, van den Bussche, Morton y Mares, 2005; Carvalho, Oliveira y Mattevi, 2009; Flores, Díaz y Bárquez, 2000; Formoso, Udrizar-Sauthier, Teta y Pardiñas, 2011; Giarla y Jansa, 2014; Giarla, Voss y Jansa, 2010; Martín, 2009; Meynard, Palma y Rivera-Milla, 2002; Palma et al., 2014; Solari, 2003; Teta, D’elía, Flores y de la Sancha, 2009), los 


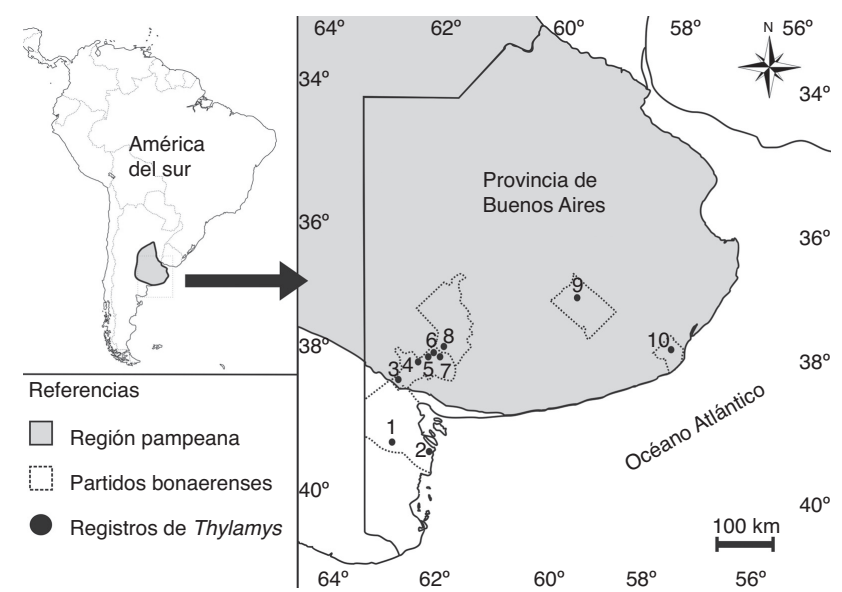

Figura 1. Mapa de la distribución geográfica de Thylamys en la Región pampeana de Argentina con detalle de su distribución en la provincia de Buenos Aires. Referencias: 1-2, Partido de Villarino (Creighton y Gardner, 2007; Martín, 2009); 3-7, Partido de Tornquist (Braun et al., 2010; Creighton y Gardner, 2007); 8, Partido de Coronel Suárez (Fernández et al., 2012); 9, Partido de Tandil (Fernández et al., 2012), y 10, Partido de General Pueyrredón (este estudio).

límites de las especies y sus áreas geográficas aún presentan importantes interrogantes. En la Argentina existen al menos entre 5 y 6 especies de Thylamys (Braun et al., 2005; Creighton y Gardner, 2007). Estudios sistemáticos y biogeográficos han demostrado que algunas de las especies corresponden realmente a complejos taxonómicos y, sobre esta base, se han descrito nuevas especies, algunas de ellas endémicas (Martín, 2009; Palma et al., 2014; Teta et al., 2009). Tanto es así que para algunas regiones de Argentina aún no existe consenso en el reconocimiento de las especies.

Al igual que en otras áreas de su distribución, una parte de las especies de Thylamys encontradas en Argentina presentan asociación con ambientes áridos (Albanese y Ojeda, 2012). Sin embargo, existen varios registros de estas comadrejas en otro tipo de ambientes, como es el caso de la región pampeana. Esta región se caracteriza por presentar extensas llanuras cubiertas por pastizales, solo interrumpidos por 2 sistemas de sierras de baja altura (Viglizzo, Frank y Carreño, 2006). Los escasos registros de Thylamys para la región corresponden a ejemplares capturados en ambientes serranos, cuya entidad taxonómica es tema de debate. Martín (2009), basándose en las características craneales indica que estos individuos pertenecen a una nueva especie endémica, Thylamys fenestrae, mientras que estudios posteriores basados en técnicas moleculares consideran que estas diferencias no serían definitivas, por lo que sugieren que fenestrae representaría una subespecie de Thylamys pallidior (T. pallidior) (Giarla et al., 2010; Palma et al., 2014).

Como parte de un relevo faunístico realizado en el sistema serrano de Tandilia (fig. 1), particularmente en sierra de los Padres (Partido de General Pueyrredón, provincia de Buenos Aires, Argentina), registramos un individuo de Thylamys sp. en abril de 2014 (fig. 2a). El mismo fue capturado a mano aproximadamente a las 13:30 h cuando se encontraba inactivo debajo de una roca rodeada por matas de Stipa sp. y Paspalum quadrifarium (fig. 2b), en un punto situado sobre la ladera de una formación serrana en la estancia «El Abrojo» (37 55'37” S;

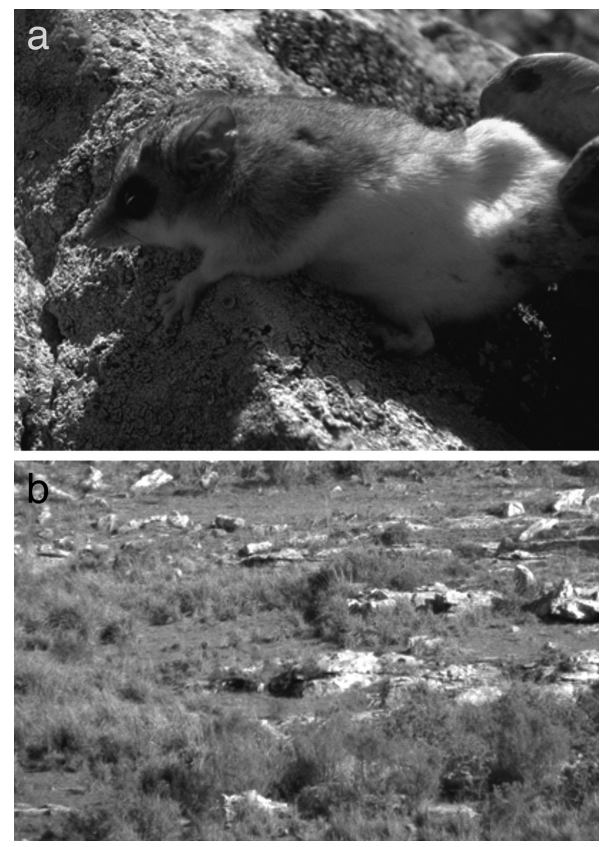

Figura 2. Foto de Thylamys sp. (a) y del sitio de captura (b) en sierra de Los Padres, Partido de General Pueyrredón, provincia de Buenos Aires, Argentina.

$\left.57^{\circ} 49^{\prime} 26^{\prime \prime} \mathrm{O}\right)$. El individuo fue fotografiado y se le tomaron medidas corporales básicas utilizando un calibre digital $(0.001 \mathrm{~mm})$, se registró el peso mediante una balanza digital $(0.1 \mathrm{~g})$, y se determinó el sexo de acuerdo con los caracteres externos (Gurnell y Flowerdew, 1990). Finalmente, el ejemplar fue liberado en el sitio de captura; se procedió a su clasificación taxonómica con base en caracteres morfológicos externos utilizando claves dicotómicas y bibliografía (Braun, Pratt y Mares, 2010; Gardner, 2007; Martín, 2009).

El ejemplar correspondió a una hembra, cuyas medidas fueron: longitud de cuerpo: $86.9 \mathrm{~mm}$; longitud de cola: $92.5 \mathrm{~mm}$; peso: $14.5 \mathrm{~g}$. Se trató de un ejemplar relativamente pequeño considerando que en este género el peso de una hembra adulta puede alcanzar $30 \mathrm{~g}$, y la longitud de cuerpo y cola puede llegar a 147 y $161 \mathrm{~mm}$, respectivamente (Creighton y Gardner, 2007). En cuanto a la coloración del individuo, la zona dorsal presentó un tono pálido grisáceo y la zona ventral un color blanco nieve, lo cual concuerda con descripciones previas para T. pallidior (Braun et al., 2010; fig. 2a). T. pallidior es, además, la especie con distribución más cercana a nuestra área de estudio y la única presente en la región pampeana (Creighton y Gardner, 2007; Palma et al., 2014), por lo que parece factible que se trate de un ejemplar de dicha especie. Sin embargo, dado que ciertas especies de Thylamys no pueden determinarse con certeza solamente por características exosomáticas, como coloración y medidas corporales, se requerirán estudios de caracteres craneométricos y/o análisis genéticos sobre la población para identificar la especie.

El presente registro amplía la distribución del género Thylamys en la región pampeana de Argentina, extendiendo el límite ca. $135 \mathrm{~km}$ hacia el E desde el registro más próximo citado hasta el momento, correspondiente a T. pallidior, para el centro de la provincia de Buenos Aires (Partido de Tandil; 37 ${ }^{\circ} 19^{\prime}$ S; 59 $8^{\prime}$ O; Fernández et al., 2012), siendo el segundo registro de este género 
para el área de las sierras de Tandilia. Los restantes registros de Thylamys para la región pampeana corresponden todos a las cercanías de la formación de sierras de Ventania (sierra de la Ventana y Laguna Chasicó, Partido de Tornquist; 38 $06^{\prime}$ S; 62 ${ }^{\circ} 13^{\prime}$ O; Braun et al., 2010; Creighton y Gardner, 2007; Martín, 2009; Pardiñas, Abba y Merino, 2004), unos $350 \mathrm{~km}$ al $\mathrm{O}$ del sitio de captura de nuestro ejemplar (fig. 1). Además, existen otros 2 registros de este género al sur de la provincia de Buenos Aires, fuera de lo que Viglizzo et al. (2006) consideran la región pampeana (Creighton y Gardner, 2007; Martín, 2009; Partido de Villarino; fig. 1). Los sistemas de Tandilia y Ventania corresponden a las 2 formaciones serranas de la provincia de Buenos Aires, donde el clima es subhúmedo a húmedo mesotermal con escasa o nula deficiencia de agua (Parker y Violante, 1989). El sistema de Ventania está conformado por sierras de mayor antigüedad y se ubica en la subregión de la Pampa Austral, mientras que el sistema de Tandilia se ubica en la subregión de Pampa Húmeda, ambos correspondientes a la provincia fitogeográfica pampeana (Cabrera, 1976). Estos sistemas serranos presentan una estructura y composición vegetal bastante similar, conformado por flechillares y pajonales (Stipa sp., Poa sp., Briza sp., Paspalum sp.) y arbustos (Baccharis sp., Colletia sp., Discaria sp.; Burkart, Bárbaro, Sánchez y Gómez, 1999; Cabrera, 1976). En este sentido, resulta interesante que todos los registros de Thylamys en la región pampeana correspondan a individuos de T. pallidior encontrados en ambientes subhúmedos serranos, lo cual contrasta con las condiciones de aridez o subaridez presentes en otras regiones de la distribución de esta especie (Albanese y Ojeda, 2012). Estudios basados en un número suficiente de individuos serían importantes para determinar tanto su entidad taxonómica como aspectos básicos de su biología en esta región marginal de su distribución.

Agradecemos a E. González-Sugasti el facilitar el acceso al sitio de muestreo. Este trabajo fue financiado por el Consejo Nacional de Investigaciones Científicas y Técnicas (Conicet) y la Universidad Nacional de Mar del Plata (EXA 15/E493).

\section{Referencias}

Albanese, S. y Ojeda, R. A. (2012). Habitat use by a Neotropical desert marsupial (Thylamys pallidior): A multi-scale approach. Mammalian Biology, 77, 237-243.

Braun, J. K., Pratt, N. L. y Mares, M. A. (2010). Thylamys pallidior (Didelphimorphia: Didelphidae). Mammalian Species, 42, 90-98.

Braun, J. K., van den Bussche, R. A., Morton, P. K. y Mares, M. A. (2005). Phylogenetic and biogeographic relationships of mouse opossums Thylamys (Didelphimorphia, Didelphidae) in Southern South America. Journal of Mammalogy, 86, 147-159.

Burkart, R., Bárbaro, N., Sánchez, R. y Gómez, D. A. (1999). Eco-regiones de la Argentina. Buenos Aires: Administración de Parques Nacionales.

Cabrera, A. L. (1976). Regiones fitogeográficas argentinas. Buenos Aires: Enciclopedia Argentina de Agricultura y Jardinería. ACME.
Carvalho, B. A., Oliveira, L. F. B. y Mattevi, M. S. (2009). Phylogeny of Thylamys (Didelphimorphia Didelphidae) species, with special reference to Thylamys karimii. Iheringia Série Zoologia, 99, 419-425.

Creighton, G. K. y Gardner, A. L. (2007). Genus Thylamys. En A. L. Gardner (Ed.), Mammals of South America, volume 1: marsupials, xenarthrans, shrews, and bats (pp. 107-117). Chicago: Chicago University Press.

Fernández, F. J., Idoeta, F., García-Esponda, C., Carrera, J. D., Moreira, G. J., Ballejo, F., et al. (2012). Small mammals (Didelphimorphia, Rodentia and Chiroptera) from Pampean Region, Argentina. CheckList, 8, 130-134.

Flores, D. A., Díaz, M. M. y Bárquez, R. M. (2000). Mouse opossums (Didelphimorphia, Didelphidae) of Northwestern Argentina: Systematics and distribution. Zeitschrift für Saugetierkunde, 65, 1-19.

Formoso, A. E., Udrizar-Sauthier, D. E., Teta, P. y Pardiñas, U. F. J. (2011). Dense-sampling reveals a complex distributional pattern between the southernmost marsupials Lestodelphys and Thylamys in Patagonia, Argentina. Mammalia, 75, 371-379.

Gardner, A. L. (2007). Mammals of South America, volume 1: marsupials, xenarthrans, shrews and bats. Chicago: Chicago University Press.

Giarla, T. y Jansa, S. (2014). The role of physical geography and habitat type in shaping the biogeographical history of a recent radiation of Neotropical marsupials (Thylamys: Didelphidae). Journal of Biogeography, 41, $1547-1558$.

Giarla, T. C., Voss, R. S. y Jansa, S. (2010). Species limits and phylogenetic relationships in the didelphid marsupial genus Thylamys based on mitochondrial DNA sequences and morphology. Bulletin of the American Museum of Natural History, 346, 1-67.

Gurnell, J. y Flowerdew, J. R. (1990). Live trapping small mammals: A practical guide. Occasional Publications of the Mammal Society of London, 3, $1-39$.

Martín, G. M. (2009). Sobre la identidad de Thylamys (Marsupialia, Didelphidae) del oeste pampeano y centro-sur del Espinal, Argentina. Mastozoología Neotropical, 16, 333-346.

Meynard, A. P., Palma, R. E. y Rivera-Milla, E. (2002). Filogeografía de las llacas chilenas del género Thylamys (Marsupialia Didelphidae) en base a secuencias del gen mitocondrial citocromo b. Revista Chilena de Historia Natural, 75, 299-306.

Palma, R. E., Boric-Bargetto, D., Jayat, J., Flores, D. A., Zeballos, H., Pacheco, V., et al. (2014). Molecular phylogenetics of mouse opossums: New findings on the phylogeny of Thylamys (Didelphimorphia Didelphidae). Zoologica Scripta, 43, 217-234.

Pardiñas, U. F. J., Abba, A. M. y Merino, M. L. (2004). Micromamíferos (Didelphimorphia y Rodentia) del sudoeste de la provincia de Buenos Aires (Argentina): taxonomía y distribución. Mastozoología Neotropical, $11,211-232$.

Parker, G. y Violante, R. A. (1989). Geología y geomorfología. Regiones I y II. Punta Rasa-Faro Querandí. Provincia de Buenos Aires. Ciudad de Buenos Aires: Convenio Consejo Federal de Inversiones y Servicio de Hidrografía Naval.

Solari, S. (2003). Diversity and distribution of Thylamys (Didelphidae) in South America with emphasis on species from the Western side of the Andes. En M. Jones, C. Dickman y M. Archer (Eds.), Predators with pouches: The biology of carnivorous marsupials (pp. 82-101). Melbourne: SCIRO Publishing.

Teta, P., D'elía, G., Flores, D. y de La Sancha, N. (2009). Diversity and distribution of the mouse opossums of the genus Thylamys (Didelphimorphia, Didelphidae) in northeastern and central Argentina. Gayana, 73, 180-199.

Viglizzo, E. F., Frank, F. C. y Carreño, L. (2006). Situación ambiental en las ecorregiones Pampa y Campos y Malezales. En A. D. Brown, U. MartínezOrtiz, M. Acerbi y J. Corcuera (Eds.), Situación ambiental argentina 2005 (pp. 263-273). Buenos Aires: Fundación Vida Silvestre. 